\title{
The future of intraoperative blood pressure management
}

\author{
Frederic Michard ${ }^{1} \cdot$ Ngai Liu $^{2} \cdot$ Andrea Kurz $^{3}$
}

Received: 14 January 2017 / Accepted: 22 January 2017 / Published online: 7 February 2017

(C) Springer Science+Business Media Dordrecht 2017

In this issue of the Journal, Rinehart et al. [1] described a new closed loop system designed to maintain blood pressure (BP) within a predetermined target zone by triggering the automatic administration of a vasopressor. This was a computer or "in silico" study where the goal was to maintain the virtual mean arterial pressure of virtual patients between 65 and $75 \mathrm{mmHg}$. In this well controlled experimental setting, the controller performance was excellent. This study was a first development step, or engineering requirement. Animal and human studies are now needed to investigate the safety and reliability of the system in real physiologic or pathophysiologic conditions, and in an environment where artifacts and measurement errors may occur. Other groups have already conducted in vivo evaluations of closed loop systems for BP control either with fluids or vasopressors [2, 3]. In 222 women having spinal anesthesia for caesarean delivery, Ngan Kee et al. [3] reported a better BP control, with fewer interventions and less wobble, with computer-controlled than with manual-controlled phenylephrine infusion. Given the exponential development of computer capabilities and digital health solutions, closed loop hemodynamic systems may become available soon in operating rooms and intensive care units $[4,5]$.

Optimal BP values remain a subject of debate in critically ill patients [6]. In surgical patients, a large $(57,315$

Frederic Michard

frederic.michard@bluewin.ch

1 MiCo, Chemin de Chapallaz 4, Denens, Switzerland

2 Department of Anesthesia, Hôpital Foch, 40 rue Worth, 92151 Suresnes, France

3 Department of General Anesthesiology, Department of Outcomes Research, Anesthesiology Institute, Cleveland Clinic, Cleveland, OH, USA patients) and recent study from the Cleveland Clinic [7] showed that the risk for postoperative myocardial and kidney injury significantly increases below a mean arterial pressure of $65 \mathrm{mmHg}$, and that a few minutes under that threshold might be deleterious. A strong relationship between the occurrence/duration of intraoperative hypotension and postoperative complications such as stroke, acute kidney injury, cardiac arrhythmia and myocardial injury is now well established [8-10]. Therefore, in the surgical context, systems monitoring BP continuously are desirable first to detect hypotension without any delay. Then, systems able to respond quickly and automatically to hypotensive events may have value to minimize their duration and potential impact on outcome, especially as very short durations of hypotension seem to be deleterious.

\section{Detecting hypotensive events during surgery}

Brachial cuffs are commonly used to measure BP in patients undergoing low to medium-risk surgery and in some patients undergoing high-risk surgery (typically hip fracture in elderly patients, colorectal resection for cancer). They allow non-invasive, intermittent measurements of BP, so that hypotension may be missed or detected with delay. In patients undergoing $3 \mathrm{~h}$ abdominal or orthopedic procedures, Chen et al. [11] showed that monitoring BP continuously has the potential, when compared to 5 min-intervals brachial cuff measurements, to decrease the duration of hypotensive events by around $20 \mathrm{~min}$. Arterial lines allow continuous BP monitoring but are associated with thrombotic, hemorrhagic and infectious complications. Therefore, they are reserved for procedures at highrisk of hemodynamic instability such as cardio-vascular, major abdomino-pelvic, and spine surgeries. Thanks to 
recent improvements in applanation tonometry [12] and volume clamp methods $[13,14]$, continuous BP monitoring is now possible non-invasively. Validation studies have yielded conflicting results; a better accuracy was reported during surgery than in ICU patients with shock. A recent meta-analysis from Cannasson's group [15] showed that these methods do not meet standards from the Association for the Advancement of Medical Instrumentation (AAMI). However, one must keep in mind that AAMI criteria have been developed to test the accuracy and precision of brachial cuffs, not alternative methods measuring continuously finger or radial BP. Interestingly, a recent study, using invasive radial measurements as the reference, reported a better precision with the volume clamp method than with the brachial cuff [16].

In the future, thanks to flexible electronics and chemical innovations, we will likely use sensors able to "feel" our pulse and record high quality blood pressure curves [17-19]. Today, these still experimental sensors are small $\left(\sim 1 \mathrm{~cm}^{2}\right)$, lightweight, thin and capable of stretching to conform to the skin, while providing high levels of pressure sensitivity and fast response time [19]. A very recent study published in Science [20] showed that adding graphene to a silicone polymer (also known as "Silly Putty" in toy stores) enables the development of super sensitive electromechanical sensors able to detect the footsteps of a small spider. When placed on the surface of our skin, next to a beating carotid artery, these sensors can record central pressure signals [19-21]. Central BP signals are more reliable than peripheral signals (e.g. radial) in the presence of peripheral decoupling, a phenomenon often associated with vasoplegia [22, 23]. Central BP signals should also enable a more accurate estimation of cardiac output by pulse contour algorithms because, unlike peripheral signals, they are almost identical to aortic signals [24].

\section{Managing hypotensive events during surgery}

In their introduction, Rinehart et al. [1] mentioned that "nearly all packaged food is the result of specially designed robots performing programmed, automated tasks". They added that "automation in medicine, however, has found limited implementation despite more than 40 years of research". Could it be because there are not two patients alike and our therapeutic decisions cannot be calibrated as packaged food? Individualized hemodynamic management is desirable and part of a larger trend called personalized or precision medicine [25, 26]. The closed loop system tested by Rinehart et al. [1] was designed to automatically administer vasopressors when the BP dropped below a predetermined value. This approach makes perfect sense in case of vasodilation-induced hypotension. However, it would not make sense in case of hypovolemia or cardiac dysfunction, and this limitation was clearly acknowledged by the authors. Understanding the underlying mechanisms of hypotension is key to ensure the correct therapy (Fig. 1). Ultimately, the clinical value of automatic systems will depend more on their programmed treatment algorithm than on the automation process itself. The former is a medical challenge (identifying the right answer to a clinical problem), the latter is a technical challenge that Rinehart et al. [1] successfully faced in their simulation study.

When used in combination with pulse contour algorithms, continuous blood pressure waveforms enable continuous monitoring of cardiac output and estimation of vascular tone (TVR), the two determinants of mean arterial pressure. When vasodilation is the cause for hypotension it may be wise to decrease anesthetic drugs instead of using vasopressors [27, 28], assuming the depth of anesthesia permits. Further analysis of the BP tracing enables the estimation of the arterial pulse pressure variation (PPV) induced by mechanical ventilation, a predictor of fluid responsiveness useful in the decision-making process of fluid administration [29].

The bottom line is that several variables and their integration are needed to develop physiology-based decision trees, mimic clinician's reasoning (Fig. 1), and envision the development of decision support tools (suggesting diagnosis and treatment) or automated systems (delivering therapy automatically). To quote Rinehart et al. in a previous

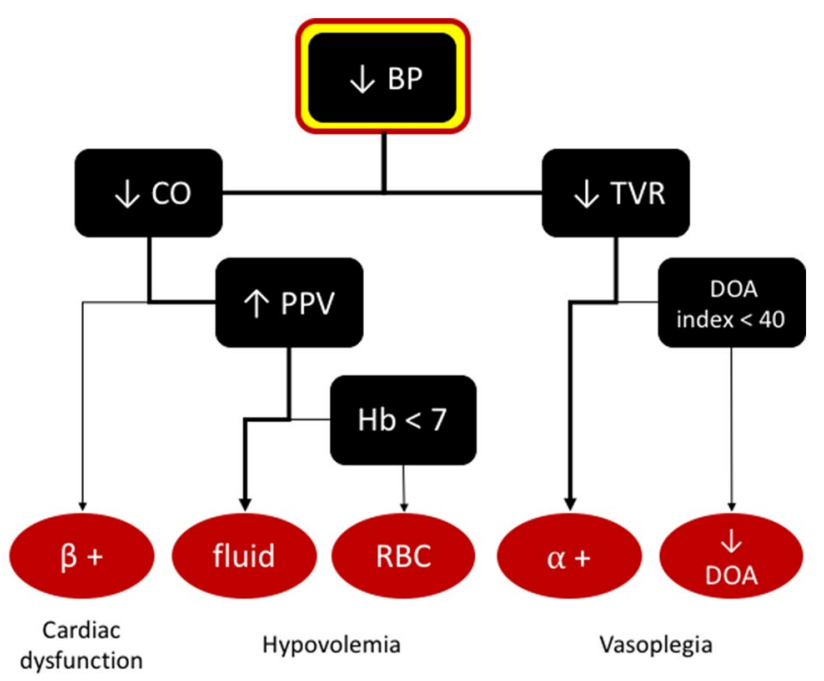

Fig. 1 Classical physiology-based reasoning in case of intraoperative hypotension. Data integration helps to make the right therapeutic decision. Bold arrows indicate the most common, but not exclusive, scenarios. All parameters (black rectangles) can now be measured continuously and non-invasively. $B P$ blood pressure, $C O$ cardiac output, $T V R$ total vascular resistance (Mean arterial pressure/CO), $P P V$ pulse pressure variation, $D O A$ depth of anesthesia, $H b$ hemoglobin, $\beta+$ inotrope, $R B C$ red blood cell, $\alpha+$ vasopressor 
publication [30], "unlike anesthetic closed loops that target depth of anesthesia [31], hemodynamic management does not lend itself to management using only a single feedback variable. These challenges will, in all probability, require sophisticated controller algorithms to manage safely".

In patients monitored with an arterial catheter, future closed loop systems for BP control will have to take into account artifacts and possible damping phenomena and zeroing issues, leading to an over or underestimation of BP. The lack of sensor redundancy may also be problematic [4]. Indeed, multiple sensors are classically needed to guarantee the quality of signals triggering automatic interventions. In airplanes, multiple airspeed sensors (or pitot tubes) are used to regulate jet engine power. While we do not insert two or more invasive arterial catheters in our patients, we most certainly can envision the simultaneous use of several non-invasive sensors to optimize BP measurements reliability, decrease false alarms, and settle the above-mentioned redundancy issue.

In the future, new materials (e.g. viscoelastic graphene polymer nanocomposites) and products (e.g. electronic tatoos) will allow us to monitor central BP and its determinants continuously and non-invasively in the operating room and beyond (in the ICU but also in ambulatory patients) [5]. Thanks to the development of connectivity between monitoring tools, data integration (Fig. 1) should become ubiquitous and useful to develop decision support tools which suggest diagnosis (e.g. vasodilation) and treatments (e.g. vasopressors). In this context, clinical studies will be required to clarify if systems delivering hemodynamic therapy automatically (with closed or open loops) are needed to further improve quality of care.

\section{Compliance with ethical standards}

Conflict of interest FM has been a consultant to Pulsion Medical Systems. and UP-MED, and an employee of Edwards Lifesciences (VP Global Medical Strategy). He is co-inventor of nine patents owned by Pulsion, UP-MED and Edwards, but does not receive any royalties. He is the founder and managing director of MiCo Sarl, a Swiss consulting firm providing services to medtech companies, digital health startups and lifescience investors.NL is co-founder of MedSteer, a biomedical company promoting research and development for closed-loop anesthesia systems.

\section{References}

1. Rinehart J, Ma M, Calderon MD, Cannesson M. Feasability of automated titration of vasopressor infusion using a novel closed loop controller. J Clin Monit Comput. 2017. doi:10.1007/ s10877-017-9981-6.
2. Kramer GC, Kinsky MP, Prough DS, et al. Closed-loop control of fluid therapy for treatment of hypovolemia. J Trauma. 2008;64:S333-41.

3. Ngan Kee WD, Khaw KS, Ng FF, Tam YH. Randomized comparison of closed-loop feedback computer-controlled with manual-controlled infusion of phenylephrine for maintaining arterial pressure during spinal anaesthesia for caesarean delivery. Br J Anaesth. 2013;110:59-65.

4. Michard F. Decision support for hemodynamic management: from graphical displays to closed loops. Anesth Analg. 2013;117:876-82.

5. Michard F, Pinsky MR, Vincent JL. Intensive care medicine in 2050: NEWS for hemodynamic monitoring. Intensive Care Med. 2017. doi:10.1007/s00134-016-4674-z.

6. Asfar P, Meziani F, Hamel JF, et al. High versus low bloodpressure target in patients with septic shock. N Engl J Med. 2014;370:1583-93.

7. Salmasi V, Maheshwari K, Yang D, et al. Relationship between intraoperative hypotension, defined by either reduction from baseline or absolute thresholds, and acute kidney and myocardial injury after noncardiac surgery. A retrospective cohort analysis. Anesthesiology. 2017;126:47-65.

8. Bijker JB, Persoon S, Peelen LM, et al. Intraoperative hypotension and perioperative stroke after general surgery. Anesthesiology. 2012;116:658-64.

9. Sun LY, Wijeysundera DN, Tait GA, Beattie WS. Association of intraoperative hypotension with acute kidney injury after elective noncardiac surgery. Anesthesiology. 2015;123:515-23.

10. Walsh M, Devereaux PJ, Garg AX, et al. Relationship between intraoperative mean arterial pressure and postoperative outcomes after noncardiac surgery: toward an empirical definition of hypotension. Anesthesiology. 2013;119:507-15.

11. Chen G, Chung E, Meng L, et al. Impact of non-invasive and beat-to-beat arterial pressure monitoring on intraoperative hemodynamic management. J Clin Monit Comput. 2012;26:133-40.

12. Meidert AS, Huber W, Muller JN, et al. Radial artery applanation tonometry for continuous non-invasive arterial pressure monitoring in intensive care unit patients: comparison with invasively assessed radial arterial pressure. Br J Anaesth. 2014;112:521-8.

13. Martina JR, Westerhof BE, van Goudoever J, et al. Non-invasive continuous blood pressure monitoring with Nexfin. Anesthesiology. 2012;116:1092-103.

14. Wagner JY, Negulescu I, Schofthaler M, et al. Continuous noninvasive arterial pressure measurement using the volume clamp method: an evaluation of the CNAP device in intensive care unit patients. J Clin Monit Comput. 2015;29:807-13.

15. Kim SH, Lilot M, Sidhu KS, et al. Accuracy and precision of continuous noninvasive arterial pressure monitoring compared with invasive arterial pressure: a systematic review and metaanalysis. Anesthesiology. 2014;120:1080-97.

16. Vos JJ, Poterman M, Mooyaart EA, et al. Comparison of continuous non-invasive finger arterial pressure monitoring with conventional intermittent automated arm arterial pressure measurement in patients under general anaesthesia. $\mathrm{Br} \mathrm{J}$ Anaesth. 2014;113:67-74.

17. Michard F. A sneak peek into digital innovations and wearable sensors for cardiac monitoring. J Clin Monit Comput. 2016. doi:10.1007/s10877-016-9925-6.

18. Schwartz G, Tee BCK, Mei J, et al. Flexible polymer transistors with high pressure sensitivity for application in electronic skin and health monitoring. Nat Commun. 2013;4:1859.

19. Dagdeviren C, Su Y, Joe P, et al. Conformable amplified lead zirconate titanate sensors with enhanced piezoelectric response for cutaneous pressure monitoring. Nat Commun. 2014;5:4496. 
20. Boland CS, Khan U, Ryan G, et al. Sensitive electromechanical sensor using viscoelastic graphene-polymer nanocomposites. Science. 2016;354:1257-60.

21. State of the art sensors using graphene and silly putty. https://

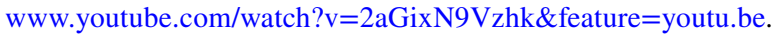
Accessed 14 Jan 2017.

22. Dorman T, Breslow MJ, Lipsett PA. Radial artery pressure monitoring underestimates central arterial pressure during vasopressor therapy in critically ill patients. Crit Care Med. 1998;26:1646-9.

23. Hatib F, Jansen JR, Pinsky MR. Peripheral vascular decoupling in porcine endotoxic shock. J Appl Physiol. 2011;111:853-60.

24. Michard F. Hemodynamic monitoring in the era of digital health. Ann Intensive Care. 2016;6:15.

25. Kato R, Pinsky MR. Personalizing blood pressure management in septic shock. Ann Intensive Care. 2015;5:41.

26. Correa TD, Jakob SM, Takala J. Arterial blood pressure targets in septic shock: is it time to move to an individualized approach? Crit Care. 2015;19:264.
27. Tsutsui T, Arita S. Fuzzy logic control of blood pressure through enflurane anesthesia. J Clin Monit. 1994;10:110-7.

28. Zbinden AM, Feigenwinter P, Petersen-Felix S, Hacisalihzade S. Arterial pressure control with isoflurane using fuzzy logic. Br J Anaesth. 1995;74:66-72.

29. Michard F. Changes in arterial pressure during mechanical ventilation. Anesthesiology. 2005;103:419-28.

30. Rinehart J, Ngai L, Alexander B, Cannesson M. Closed-loop systems in anesthesia: is there a potential for closed-loop fluid management and hemodynamic optimization? Anesth Analg. 2012;114:130-43.

31. Liu N, Le Guen M, Benabbes-Lambert F, et al. Feasability of closed-loop titration of propofol and remifentanil guided by the spectral M-Entropy. Anesthesiology. 2012;116:286-95. 